\title{
Tadalafil once daily: Narrative review of a treatment option for female sexual dysfunctions (FSD) in midlife and older women
}

\author{
Chiara Borghi ${ }^{1}$, Lucio Dell'Atti ${ }^{2}$ \\ ${ }^{1}$ Department of Morphology, Surgery and Experimental Medicine, Institute of Obstetrics and Gynecology, University of Ferrara, Italy; \\ ${ }^{2}$ Department of Urology, S. Anna University Hospital, Ferrara, Italy.
}

\begin{abstract}
Summary Female Sexual Disorders (FSD) include a complex, multidimensional, individual experience that can change as an individual age, suggesting that these problems are caused by multiple factors including psychosocial factors, personal relationships, pathologic changes caused by diseases, and pharmacologic influences. Menopause is an important time for middle aged women and postmenopausal physiological changes could have a significant role in the development of FSD. Few is still known about their correct definition and treatment. Their incidence, prevalence and risk factors are difficult to define because of a high level of overlap in the experience of problems with desire, arousal, and orgasm. Little evidences are known about the best therapeutic approach, and both non-pharmacological and pharmacological treatment options have been described. Among these, phosphodiesterase type 5 inhibitors could be an effective option for many subtypes of female sexual disorders, with an improvement in different aspects of sexual function, such as desire, arousal, orgasm and sexual satisfaction. In this paper authors reviewed what is already known about the use of these vasoactive agents, particularly tadalafil, as a treatment option for female sexual disturbances.
\end{abstract}

KEY WORDS: Female Sexual Disorders; Phosphodiesterase type 5 inhibitors; Tadalafil; Women.

Submitted 31 August 2016; Accepted 24 December 2016

\section{INTRODUCTION}

Sexual and reproductive health and well-being are essential to guarantee responsible, safe and satisfying sexual lives, thus requiring an understanding of the complex factors that regulate human sexual behaviour. According to the World Health Organization (WHO) revised definition, sexual health is a state of physical, emotional, mental, and social wellbeing, underpinned by human rights, in relation to sexuality $(1,2)$.

Female Sexual Dysfunctions (FSD) are high prevalent, age-related and progressive problems, whose definition is complicated, and significantly less is understood in comparison to male sexual dysfunction. The sexual response cycle in women is done by complex interactions, psychological, social and biologic factors (hormonal, vascular and neuromuscular). Menopause is an important time for middle aged women and postmenopausal physiological changes could have a significant role in the development of FSD. Nevertheless, despite an increased awareness of its pathophysiology, studies didn't find an agreement on what is the best therapeutic approach and currently there are no drugs approved for most of the complaints (3).

Phosphodiesterase type 5 inhibitors (PDE5i) have been widely used for the treatment of male sexual dysfunction (4). In this paper authors review the current literature on FSD and analyse PDE5i, particularly tadalafil, as a treatment option for this health issue.

\section{Current knowledge of female seXual dysfunction}

The concept of FSD comprises a wide range of disorders, whose definition has undergone numerous changes and classifications in the past. Different definitions included hypoactive sexual desire, impaired subjective or physical genital arousal, sexual pain and inability to achieve orgasm, which are multidimensional issues, often coexisting. The most frequently used classification systems are the International Classification of Diseases, $10^{\text {th }}$ Edition (ICD-10) by the WHO (5) and the Diagnostic and Statistical Manual of Mental Disorder, Fifth Edition (DSM-5) by the American Psychiatric Association (6). The former subdivides the disorders as organic or non organic. For female, organic disorders include vaginismus and dyspareunia of organic aetiology. Non organic female sexual dysfunctions include lack of sexual desire, sexual aversion or lack of sexual enjoyment, failure of genital response, orgasmic dysfunction, non-organic vaginismus, non-organic dyspareunia, excessive sexual drive and two non-specific codes. These causes are summarized in Table 1. The latter, introduced in 2013, defines the most prevalent subcategory of FSD with a global approach, namely female sexual interest/arousal disorders. The second group is called female orgasmic disorder, which includes marked delay in, marked infrequency of, or absence of orgasm and decreased intensity of orgasmic sensations. The other important branch of sexual pain disorders (vaginismus and dyspareunia) is also renamed into genito-pelvic pain/penetration disorders. According to this classification system, the diagnosis of a sexual dysfunction (except substance/medication-induced sexual dysfunction) requires a duration of symptoms of at least six months and an occur- 
Table 1.

Organic and Non Organic causes of FSD, according to $I C D-10 *$.

\begin{tabular}{|l|l|}
\hline Organic sexual dysfunctions & Nonorganic sexual dysfunctions \\
\hline Vaginismus of organic etiology & Lack of sexual desire \\
\hline Dyspareunia of organic etiology & Sexual aversion \\
\hline & Lack of sexual enjoyment \\
\hline & Failure of genital response \\
\hline & Orgasmic dysfunction \\
\hline & Non-organic vaginismus \\
\hline & Non-organic dyspareunia \\
\hline *ICD-10: International Classification of Diseases, $10^{\text {th }}$ Edition. \\
\hline
\end{tabular}

rence in approximately $75 \%$ of sexual occasions. The classification of FSD according to DSM-5 is summarised in Table 2. Further sub-classification includes lifelong versus acquired and generalized versus situational types (6).

The literature on the incidence, prevalence and risk factors for female sexual dysfunction indicates a high level of overlap in the experience of problems with desire, arousal, and orgasm for women. Because of these wide features, there are inherent difficulties in collection of data for epidemiological studies that can be significantly linked to clinically based definitions and classifications. Furthermore, the lacking available literature is influenced by difficulties in comparing findings that include different recording systems and characteristics of the involved population in terms of age, cultural and socioeconomic background $(7,8)$.

However, the most important epidemiological studies in the past found that FSD is considered a major complaint among women, involving approximately $43 \%$ of female population worldwide (9).
Its incidence ranges from $5.8 \%$ to $45 \%$ in different studies and percentages are higher in older women subgroups (20\% in women younger than 25 and $70-80 \%$ in women aged between 55 and 74). The most common dysfunction is hypoactive sexual desire (25-45\%), followed by low arousal (11-26\%) and orgasm difficulties (11-21\%). Lower percentages of women reported combined symptoms. Noticeably, females report sexual disorders more frequently than men (43\% versus $31 \%)(8,10)$.

The prevalence (proportion of a given population that at a given time has a particular condition) of FSD also varies widely according to different age groups and countries. Low sexual interest and desire involves percentages ranging from 17 to $55 \%$ of women younger than 59 , and from 17 to $34 \%$ of women aged 40 to 80 . More recent studies report an overall prevalence rate of 40-50\% among women older than 65. Epidemiologic data report considerable differences also regarding prevalence of lubrication and arousal dysfunction (12-41.9\%), orgasmic dysfunction (11-72\%) and genito-pelvic pain (1-68\%) (8, 11-16). Sexual function in women results from a complex neurovascular process that is controlled by psychological, psychosocial, inter-personal and endocrine inputs, consequently the aetiology and risk factors of FSD can be due to one or more systems and there is no consensus on the contribution of each of them (17). Already known risk factors for FSD are of biological, psychological and socio-cultural origin and their role on the different aspects of sexual disorders is often unclear. As an example, biological factors seem to be related to disorders of desire and arousal, but their role in orgasm disorders is less clear. They include age, diabetes, heart disease, chronic health conditions, urinary tract symptoms, previous hysterectomy, female genital mutilation. Overall, perceived poor health conditions are considered as predictors for women's lack of sexual desire, arousal, orgasm and dyspareunia (7, 18-19).

\section{Table 2}

Sexual dysfunctions in women, according to DSM-5 ${ }^{\circ}$.

\begin{tabular}{|c|c|}
\hline Etiologic subtype & Symptoms, disorders, difficulties \\
\hline $\begin{array}{l}\text { Female sexual interest-arousal disorder } \\
\text { (at least three of the following characteristics) }\end{array}$ & $\begin{array}{l}\text { - absent or decreased interest in sexual activity } \\
\text { - absent or decreased sexual or erotic thoughts or fantasies } \\
\text { - absent or decreased initiation of sexual activity and unreceptive to a partner's attempts to initiate } \\
\text { - absent or decreased sexual excitement or pleasure during sexual activity in almost all or all sexual encounters } \\
\text { - absent or decreased sexual interest or arousal in response to any internal or external sexual or erotic cues } \\
\text { - absent or decreased genital or non-genital sensations during sexual activity in almost all or sexual encounters }\end{array}$ \\
\hline $\begin{array}{l}\text { Female orgasmic disorder } \\
\text { (either of the following) }\end{array}$ & $\begin{array}{l}\text { - marked delay in, marked infrequency of, or absence of orgasm } \\
\text { - markedly decreased intensity of orgasmic sensations }\end{array}$ \\
\hline $\begin{array}{l}\text { Genito-pelvic pain-penetration disorder } \\
\text { (at least one of the following difficulties) }\end{array}$ & $\begin{array}{l}\text { - vaginal penetration during intercourse } \\
\text { - marked vulvovaginal or pelvic pain during vaginal intercourse or penetration attempts } \\
\text { - marked fear or anxiety about vulvovaginal or pelvic pain in anticipation of, during, or as a result of vaginal penetration } \\
\text { - marked tensing or tightening of the pelvic floor muscles during attempted vaginal penetration }\end{array}$ \\
\hline Substance- or medication-induced sexual dysfunction & - sexual dysfunction etiologically related to a medication or substance (occurs in both men and women) \\
\hline Other specified sexual dysfunction & - other \\
\hline Unspecified sexual dysfunction & - unspecified \\
\hline
\end{tabular}


In terms of psychological and psychiatric risk factors, anxiety and depression have confirmed association with sexual dysfunction, furthermore the medication used to treat them particularly affect orgasmic function (20).

Less is known about the role of social and cultural factors on FSD but it is clear that low education, low quality of the relationship, social and working difficulties, substance abuse and traumatic life experiences have a negative influence (7).

Epidemiologic studies will be more detailed and useful if standard definitions of sexual dysfunction, validated measurement instruments and uniform description of study design will be used in order to decrease all the variations reported in the current ones.

Because of this wide range of features, the therapeutic approach on FSD cannot be univocal and, in general, little evidence is known on both non-pharmacological treatment modalities (such as psychosexual treatments) and pharmacological treatment options (21). If possible, therapy should focus on the most likely causal factor, taking into consideration the frequent overlapping between biological, relational and psychological factors. Among pharmacological possibilities, hormonal therapies with androgens, estrogens or selective estrogen receptor modulators are to be mentioned, psychotropic agents such as dopamine agonists have also been studied and herbal supplements or other nonmedical treatment have been used with unproven efficacy (22).

Interestingly, the success of vasoactive agents such as PDE5i in the treatment of male sexual arousal dysfunction have encouraged the research of therapies that enhance women's genital congestion and vaginal lubrication and PDE5i have been used in several studies about FSD, with variable results (21).

\section{MECHANISM OF ACTION \\ OF PHOSPHODIESTERASE TYPE $\mathbf{5}$ INHIBITORS}

Smooth muscle contraction and relaxation is regulated by a mechanism involving the Nitric Oxide (NO) vasodilation pathway. $\mathrm{NO}$ activates the guanylate cyclase enzyme, which converts guanosine triphosphate (GTP) into cyclic guanosine monophosphate (cGMP). cGMP is an important second messenger that plays a central role in signal transduction and regulation of many physiologic responses, such as smooth muscle tone. It is degraded by phosphodiesterase (PDE) isoenzymes, a heterogeneous group of hydrolytic enzymes. PDE5 inhibition should thus result in an increased $\mathrm{NO}$ signal and improved vascular function (23). To date, eleven different PDE families have been identified, each typically hav- ing several different isoforms and splice variants. Among these, PDE5 is specific for CGMP and PDE5i have been proved to be of pharmacological relevance, widely studied and used (24). PDE5i were originally designed to treat cardiovascular diseases and later used for the treatment of male erectile dysfunction with acceptable results (4). Other fields of application have been analysed and, in spite of several studies, the exact mechanism of action and efficacy of PDE5i in the treatment of FSD remain still unclear. PDE5 enzymes and the NO neurotransmitter mechanism have been detected in vaginal smooth muscle tissue, clitoral shaft and also in the uterine tissue and increased vaginal blood flow pulse amplitude has been detected after treatment with a PDE5i. Consequently, treatment with PDE5i can decrease the catabolism of cGMP in females, thus resulting in the relaxation of clitoral and vaginal smooth muscle and increased blood flow in these sites (25). Up to date, six oral PDE5i are commercially available, which are sildenafil, vardenafil, tadalafil, avanafil, udenafil and mirodenafil. Though success of PDE5i in males with sexual dysfunction did stimulate interest in treating FSD, the same effectiveness of this drug class has not been found across genders (17). A few studies analysed the efficacy of sildenafil as a treatment for many subtypes of FSD (26) but even less is known about the role of other molecules belonging to the PDE5i family. Newly developed compounds may contain certain advantages over sildenafil, such as higher selectivity for PDE5 compared with other isoenzymes, faster onset, longer duration of effect and absence of food effect on absorption, which consequently, allowing more flexibility in sexual activity (24).

\section{TADALAFIL}

Tadalafil appears to be as effective as sildenafil but has a longer duration of action, between 24 and 36 hours, with a mean elimination half-life of 17.5 hours, compared to the 4-5 hours elimination half-life of sildenafil. This prolonged half-life, due to its pharmacokinetic properties, lets this drug available for once daily administration. This approach appeared to be as effective as taking higher doses on an as-needed basis in men with erectile dysfunction (27). This dosing regimen has the advantage of separating administration from sexual interaction and it could me more suitable also for female population. Unlike sildenafil or vardenafil, tadalafil absorption is unaffected by food or alcohol consumption (28). Tadalafil has been widely used in both sexes for the treatment of several pathologies, particularly its efficacy has been studied in lower urinary tract symptoms $(24,29)$

\section{Table 3.}

Published studies about female sexual disorders treated with tadalafil.

\begin{tabular}{|l|c|c|c|c|}
\hline Reference & \multicolumn{1}{|c|}{ Treatment } & $\mathbf{N r}$ & FSD \\
\hline Aston et al. 2006 & Tadalafil $20 \mathrm{mg}$ on demand & 3 & $\begin{array}{c}\text { Sexual dysfunction induced by serotonin-enhancing medications } \\
\text { Lack of sexual desire. }\end{array}$ & Resolution of sexual disorders \\
\hline Caruso et al. 2012 & Tadalafil $5 \mathrm{mg}$ once daily for 12 weeks & 33 & $\begin{array}{c}\text { Premenopausal type 1 diabetic women } \\
\text { Genital arousal disorders }\end{array}$ & $\begin{array}{l}\text { Resolution of sexual disorders } \\
\mathrm{Nr}=\text { number of patients; FSD = Female Sexual Disorders. }\end{array}$ \\
\hline
\end{tabular}


for its metabolic and micro-vascular effects on skeletal muscle and adipose tissue in type 2 diabetic patients (23), for the treatment of pulmonary hypertension (30). Little evidence is reported on its efficacy in the treatment of FSD, specially in particular populations such as women treated with antidepressant drugs (31) or women affected by type 1 Diabetes Mellitus (DM) with sexual arousal disorders (28). The first study described the use of tadalafil $20 \mathrm{mg}$ prior to sexual activity by three women treated for anxiety disorder or panic disorder, who reported sexual dysfunction induced by serotoninenhancing medications. All these patients experienced resolution of the sexual disorders, and authors concluded that augmentation with tadalafil may help some women with serotonin-reuptaking inhibiting-induced sexual dysfunction (31). The second study analysed a population of 33 premenopausal type 1 diabetic women affected by sexual genital arousal disorder and treated with daily tadalafil $5 \mathrm{mg}$ administration for 12 weeks. Authors concluded that this therapeutic scheme seems to improve subjective sexual aspect in the analysed population and can be used to treat genital arousal disorder of premenopausal women with type 1 DM (28).

In both studies tadalafil administration seems to improve subjective sexual aspects in the observed population, but they noticeably have some limitations, first of all the small sample, the lack of a placebo-control group and the lack of objective evaluation of the subtype of sexual aspects involved.

\section{Conclusions}

Female sexual disorders include a complex, multidimensional, individual experience that can change as an individual age, suggesting that FSD is caused by multiple factors including psychosocial factors, personal relationships, pathologic changes caused by diseases, and pharmacologic influences. Furthermore, postmenopausal hormonal changes could have a significant role in the development of FSD. Clinicians should correctly assess patients' sexual health and provide open communication, medical understanding and education, in order to improve the medical assistance and let patients face sexual issues as they might arise.

Non pharmacologic interventions are the first choice in the approach to these disturbances, but they are often ineffective. Pharmacotherapy for treating FSD is still under investigation and, to date, no treatments have been approved by the Food and Drug Administration (32). PDE5i have been used in the treatment of erectile dysfunction with acceptable results (33). The treatment with PDE5i can decrease the catabolism of cyclic GMP also in female individuals, resulting in the relaxation of clitoral and vaginal smooth muscle, increasing local blood flow (32). Although some researchers reported negative results, several authors demonstrated that treatment with PDE5i, particularly sildenafil, could be an effective option for many subtypes of FSD if compared to placebo, with an improvement in different aspects of sexual function, such as sexual desire, sexual arousal, sexual orgasm and sexual satisfaction (26). PDE5i administration can be related to known adverse effects, for example headache, nausea, flushing or vision changes, but all of them can be quickly solved with the discontinuation of therapy, thus implying that these medications could be safe. Few is known about the use of tadalafil in this field and, since this molecule is well known and used in many different pathologies, we think that its administration should be studied deeper as a treatment option for FSD, with well designed randomized placebo controlled trials. Therefore, well-designed randomized controlled trials should be performed to report results of the treatment with PDE5i.

\section{REFERENCES}

1. Hawkes S. Sexual health: A post-2015 palimpsest in global health? Lancet Glob Heal. 2014; 2:e377-e378.

2. World Health Organization. Defining sexual health. Report of a technical consultation on sexual health. Sex Heal Doc Ser. 2002. http://www.who.int/reproductivehealth/publications/sexual_health/d efining_sexual_health.pdf

3. Belkin Z, Krapf J, Goldstein A. Drugs in early clinical development for the treatment of female sexual dysfunction. Expert Opin Investig Drugs. 2015;24:159-167.

4. Doumas M, Lazaridis A, Katsiki N, Athyros V. PDE-5 inhibitors: clinical points. Curr Drug Targets. 2015; 16:420-426.

5. World Health Organization. International statistical classification of diseases and related health problems. 10th ed. Geneva: World Health Organization. 1992. http://www.who.int/classifications/icd/en/

6. American Psychiatric Association. Diagnostic and statistical manual of mental disorders. 5th ed. Washington, DC: American Psychiatric Press. 2013. http://www.dsm5.org/Pages/Default.aspx

7. McCabe MP, Sharlip ID, Lewis R, et al. Risk Factors for Sexual Dysfunction Among Women and Men: A Consensus Statement From the Fourth International Consultation on Sexual Medicine 2015; J Sex Med. 2016; 13:153-167.

8. McCabe MP, Sharlip ID, Lewis $R$, et al. Incidence and Prevalence of Sexual Dysfunction in Women and Men: A Consensus Statement from the Fourth International Consultation on Sexual Medicine 2015. J Sex Med. 2016; 13:144-152.

9. Shifren JL, Monz BU, Russo PA, Segreti A. Sexual Problems and Distress in United States Women. 2008; 112:970-978.

10. Burri A, Spector T. Recent and lifelong sexual dysfunction in a female UK population sample: prevalence and risk factors. J Sex Med. 2011; 8:2420-2430.

11. de Visser RO, Richters J, Rissel C, et al. Change and stasis in sexual health and relationships: comparisons between the First and Second Australian Studies of Health and Relationships. Sexual Health, 2014; 11:505-509.

12. Amidu N, Owiredu W, Woode E, et al. Incidence of sexual dysfunction: a prospective survey in Ghanaian females. Reprod Biol Endocrinol. 2010; 8:106.

13. Dennerstein L, Guthrie JR, Hayes RD, et al. Sexual function, dysfunction, and sexual distress in a prospective, population-based sample of mid-aged, Australian-born women. J Sex Med. 2008; 5:2291-2299.

14. Hayes RD, Dennerstein L, Bennett CM, Fairley CK. What is the "true" prevalence of female sexual dysfunctions and does the way we assess these conditions have an impact? J Sex Med. 2008; 5:777-787. 
15. McCabe MP, Goldhammer DL. Prevalence of women's sexual desire problems: What criteria do we use? Arch Sex Behav. 2013; 42:1073-1078.

16. Safarinejadinal M. Female sexual dysfunction in a populationbased study in Iran: prevalence and associated risk factors. Int J Impot Res. 2006; 18:382-395.

17. Allahdadi KJ, Tostes RC, Clinton Webb R. Female Sexual Dysfunction: Therapeutic Options and Experimental Challenges. Cardiovasc Hematol Agents Med Chem. 2009; 7:260-269.

18. Miles CL, Candy B, Jones L, et al. Interventions for sexual dysfunction following treatments for cancer. Cochrane Database Syst Rev. 2007; CD005540.

19. Clayton AH, Harsh V. Sexual Function Across Aging. Curr Psychiatry Rep. 2016; 18:28.

20. Serretti A, Chiesa A. A meta-analysis of sexual dysfunction in psychiatric patients taking antipsychotics. Int Clin Psychopharmacol. 2011; 26:130-40.

21. Giraldi A, Rellini AH, Pfaus J, Laan E. Female Sexual Arousal Disorders. J Sex Med. 2013; 10:58-73.

22. Bonfim Reis SL, Abdo CHN. Benefits and risks of testosterone treatment for hypoactive sexual desire disorder in women : a critical review of studies published in the decades preceding and succeeding the advent of phosphodiesterase type 5 inhibitors. Clinics. 2014; 69:294-303.

23. Jansso PA, Murdolo G, Sjögren L, et al. Tadalafil increases muscle capillary recruitment and forearm glucose uptake in women with type 2 diabetes. Diabetologia. 2010; 53:2205-2208.

24. Zhang $W H$, Zhang XH. Clinical and preclinical treatment of urologic diseases with phosphodiesterase isoenzymes 5 inhibitors: an update. Asian J Androl 2016; 18: 723-731.

25. Chivers ML, Rosen RC. Phosphodiesterase Type 5 Inhibitors and Female Sexual Response: Faulty Protocols or Paradigms? J Sex Med. 2010; 7:858-872.

26. Gao L, Yang L, Qian S, et al. Systematic review and meta-analysis of phosphodiesterase type 5 inhibitors for the treatment of female sexual dysfunction. Int J Gynecol Obstet. 2016; 133:139-145.

27. Porst H, Giuliano F, Glina S, et al. Evaluation of the efficacy and safety of once-a-day dosing of tadalafil $5 \mathrm{mg}$ and $10 \mathrm{mg}$ in the treatment of erectile dysfunction: results of a multicenter, randomized, double-blind, placebo-controlled trial. Eur Urol. 2006; 50:351-359.

28. Caruso S, Cicero C, Romano M, et al. Tadalafil $5 \mathrm{mg}$ Daily Treatment for Type 1 Diabetic Premenopausal. J Sex Med. 2012; 9:2057-2065.

29. Andersson K-E, Nomiya M, Sawada N, Yamaguchi O. Pharmacological treatment of chronic pelvic ischemia. Ther Adv Urol. 2014; 6:105-114.

30. Rusiecki J, Rao Y, Cleveland J, et al. Sex and menopause differences in response to tadalafil ì: 6-minute walk distance and time to clinical worsening. Pulm Circ. 2015; 5:701-706.

31. Ashton A, Weinstein W. Tadalafil reversal of sexual dysfunction caused by serotonin enhancing medications in women. J Sex Marital Ther. 2006; 32:1-3.

32. Nappi RE. Why are there no FDA-approved treatments for female sexual dysfunction? Expert Opin Pharmacother. 2015; 16:1735-1738.

33. Hakky TS, Jain L. Current use of phosphodiesterase inhibitors in urology. Turkish J Urol. 2015; 41:88-92.

\section{Correspondence}

Chiara Borghi, $M D$

Department of Morphology, Surgery and Experimental Medicine, Institute of Obstetrics and Gynecology, University of Ferrara, Ferrara, Italy 